OPEN ACCESS

Edited by:

Faming Fang,

East China Normal University, China

Reviewed by:

Guochun Shen,

East China Normal University, China

Jun Liu,

Northeast Normal University, China

*Correspondence:

En Li

en.li@ia.ac.cn

Specialty section:

This article was submitted to Technical Advances in Plant Science,

a section of the journal

Frontiers in Plant Science

Received: 22 April 2021

Accepted: 06 September 2020

Published: 01 October 2021

Citation:

Tian Y, Li E, Liang Z, Tan M and He X (2021) Diagnosis of Typical Apple

Diseases: A Deep Learning Method

Based on Multi-Scale Dense

Classification Network.

Front. Plant Sci. 12:698474.

doi: 10.3389/fpls.2021.698474

\section{Diagnosis of Typical Apple Diseases: A Deep Learning Method Based on Multi-Scale Dense Classification Network}

\author{
Yunong Tian ${ }^{1,2}$, En Li ${ }^{1,2 *}$, Zize Liang ${ }^{1,2}$, Min Tan ${ }^{1,2}$ and Xiongkui He ${ }^{3}$ \\ ${ }^{1}$ State Key Laboratory of Management and Control for Complex Systems, Institute of Automation, Chinese Academy of \\ Sciences, Beijing, China, ${ }^{2}$ School of Artificial Intelligence, University of Chinese Academy of Sciences, Beijing, China, \\ ${ }^{3}$ College of Science, China Agricultural University, Beijing, China
}

Disease has always been one of the main reasons for the decline of apple quality and yield, which directly harms the development of agricultural economy. Therefore, precise diagnosis of apple diseases and correct decision making are important measures to reduce agricultural losses and promote economic growth. In this paper, a novel Multi-scale Dense classification network is adopted to realize the diagnosis of 11 types of images, including healthy and diseased apple fruits and leaves. The diagnosis of different kinds of diseases and the same disease with different grades was accomplished. First of all, to solve the problem of insufficient images of anthracnose and ring rot, Cycle-GAN algorithm was applied to achieve dataset expansion on the basis of traditional image augmentation methods. Cycle-GAN learned the image characteristics of healthy apples and diseased apples to generate anthracnose and ring rot lesions on the surface of healthy apple fruits. The diseased apple images generated by Cycle-GAN were added to the training set, which improved the diagnosis performance compared with other traditional image augmentation methods. Subsequently, DenseNet and Multi-scale connection were adopted to establish two kinds of models, Multi-scale Dense Inception-V4 and Multi-scale Dense Inception-Resnet-V2, which facilitated the reuse of image features of the bottom layers in the classification neural networks. Both models accomplished the diagnosis of 11 different types of images. The classification accuracy was 94.31 and $94.74 \%$, respectively, which exceeded DenseNet-121 network and reached the state-of-the-art level.

Keywords: apple disease diagnosis, Cycle-GAN, Multi-scale connection, DenseNet, deep learning

\section{INTRODUCTION AND RELATED WORKS}

Nowadays, apple is one of the most widely grown, highly productive and popular fruits in the world. The quality of fruits directly determines the economic development of the apple plantation industry. However, different kinds of diseases have always been one of the major causes for the decline in apple quality and yield, and directly harm the development of agricultural economy. Therefore, precise diagnosis of apple diseases and correct treatments are important measures to alleviate agricultural losses and promote economic development. 
At present, the diagnosis of most plant diseases still depends on farmers. However, as the image features of some diseases are similar, and there is no obvious boundary between different grades of the same disease, the artificial diagnosis results might present a large deviation. This poses a challenge to disease management. Moreover, due to the random occurrence, some diseases cannot be found in time. This will affect the quality and yield of fruits, and then harm the development of agricultural economy. Therefore, it is increasingly significant to use computer vision and deep learning methods to achieve automatic and precise disease diagnosis. With the continuous maturity of agricultural Internet of Things (IoT) technology, sensor networks and information perception systems are increasingly employed in agricultural industry (Khanna and Kaur, 2019; Muangprathub et al., 2019). This plays a key role in obtaining timely disease information and making precise decisions on disease prevention. In terms of plant disease information processing, image information has become a common basis for identifying disease types and judging the grades of the disease (Sankaran et al., 2010; Zhang J. et al., 2019).

In traditional image processing, binarization is often utilized to extract lesion areas. These methods can process images with simple backgrounds, but they cannot be readily applied in complex image background due to the lack of available image features. In the past few years, traditional image processing techniques have been widely adopted in plant disease identification and detection. Zou et al. (2010) used a multi-threshold segmentation technique to detect the lesion area of apples. This method can extract the lesion area on the surface of the apple, but the disease type cannot be diagnosed. Zarifneshat et al. (2012) adopted artificial neural network (ANN) to predict the volume of the apple lesion. Rumpf et al. (2010) made use of the support vector machine (SVM) to classify the multi-spectral images of lesions and realized the diagnosis of plant diseases. Omrani et al. (2014) used SVM and ANN to diagnose diseases such as apple spot alternaria and black spot, and compared the performance of the two models. In our previous research, a feedback neural network optimized by a genetic algorithm was employed to segment apple fruit regions in complex environments (Tian et al., 2018). The SVM method was then used to extract the lesion areas in the fruit images. This method realized the detection of apple diseases in a complex environment. Although traditional methods have achieved the diagnosis and detection of plant diseases in certain circumstances, they are not effective in complex environments. Moreover, the image processing time is relatively long, which cannot meet the demand for timely and accurate plant disease diagnosis.

With the continuous upgrading of processor computing power, artificial intelligence and machine learning technologies are favored in the field of smart agriculture (Patricio and Rieder, 2018). In terms of image processing of plant diseases, Mohanty et al. (2016) used AlexNet (Krizhevsky et al., 2012) and GoogLeNet (Szegedy et al., 2015) to realize the recognition of 14 crops and 26 diseases, with the accuracy of $99.35 \%$.
The dataset images used in this research are crop images with monotonous backgrounds, rather than taken in field. Amara et al. (2017) adopted LeNet (Lecun et al., 1998) as the backbone to identify banana leaf diseases under complex backgrounds and illumination conditions. Sladojevic et al. (2016) made use of CaffeNet (Jia et al., 2014) to classify 13 leaf diseases, and the accuracy reached $96.3 \%$. Jiang et al. (2019) proposed an INAR-SSD method for real-time diagnosis and detection of 5 common apple leaf diseases, achieving $78.80 \%$ mean average precision and 23.13 frames per second. Zhang S.et al. (2019) proposed a global pooling dilated convolution neural network to diagnose 6 types of common cucumber leaf diseases with the accuracy of $94.65 \%$, which is better than commonly used depth convolutional neural networks. Zhong and Zhao (2020) adopted DenseNet-121 (Huang et al., 2017) backbone to diagnose 6 types of apple leaf diseases. Three methods of regression, multilabel classification and attention loss function were employed for comparison on the basis of DenseNet-121. The accuracy reached 93.51, 93.31, and 93.71\% respectively, achieving the state-of-theart level. However, due to the phenomenon of image feature loss, it is difficult to improve the diagnostic accuracy merely by increasing the depth of network layers, which brings a challenge to meet the increasing demand for diagnosis.

The outstanding performance of convolutional neural networks is based on sufficient training data. During the acquisition of apple disease images, the randomness of disease occurrence makes it difficult to collect a large amount of data. Traditional image augmentation methods, such as rotation (Tan et al., 2016), mirroring (Dyrmann et al., 2016), translation (Sladojevic et al., 2016), scale transformation (Bargoti and Underwood, 2016), brightness transformation, color transformation (Tian et al., 2019b) and image mosaic (Tian et al., 2020), have been applied in the process of dataset establishment. However, these methods do not change the superpixel information of the image, and still retain the similarity of the color, brightness, texture, and other features between the adjacent pixels of the original image. In our previous research, the Cycle-GAN (Isola et al., 2018; Shen et al., 2019) method was employed to generate anthracnose lesion images on the surface of healthy apples (Tian et al., 2019a). This method effectively expanded the training set. However, in our previous work, only anthracnose images have been generated. Various kinds of diseases and different grades of the same disease need to be further processed to verify the feasibility of the algorithm.

In this paper, a Multi-scale Dense convolutional neural network backbone is designed for diagnosing 11 types of images including healthy and diseased apple fruits and leaves. The main contributions are as follows:

(1) Cycle-GAN method was adopted to generate two diseases, anthracnose and ring rot, on the surface of healthy apples. This method completed the data augmentation in the superpixel space.

(2) A Multi-scale Dense neural network backbone for apple disease diagnosis was proposed. The effectiveness of existing classification models has been improved. 
(3) It was validated in the experiment that the proposed method outperformed other common disease diagnosis models, and achieved the state-of-the-art level.

The paper has been organized in the following manner. Section 2 introduces the materials and methods, including image acquisition, image augmentation, and apple disease diagnosis models. Section 3 concerns the experiments and the performance of the proposed methodologies. Finally, our conclusions are presented.

\section{MATERIALS AND METHODS}

\subsection{Dataset Preparation}

\subsubsection{Composition of Image Dataset}

The dataset employed in our research includes diseased leaf images, diseased fruit images, healthy leaf images, and healthy fruit images, as shown in Figure 1. The images of apple leaves come from Challenger-Plant-Disease-Recognition (https:// gitee.com/cheng_xiao_yuan/AI-Challenger-Plant-Disease-

Recognition). The leaf images are divided into six categories, including healthy apple leaf, general apple scab, serious apple scab, apple gray spot, general cedar apple rust, and serious cedar apple rust. The fruit images were collected in the field. These images include five categories, including healthy green apple fruit, healthy red apple fruit, general anthracnose, serious anthracnose, and ring rot. The number of original images collected is shown in Table 1. Due to the random occurrence of apple diseases, it is arduous to obtain abundant images of diseases in the field. Therefore, the number of collected apple images of anthracnose and ring rot is far less than that of other types of diseases, which cannot meet the training requirements of deep learning neural networks. Since some of the image features of anthracnose and ring rot are similar, and there is no clear boundary between different grades of the same disease, more images are needed to obtain rich image features. However, the images collected in the field were insufficient for training the deep neural networks.

\subsubsection{Image Data Augmentation}

In order to meet the demand for the number of images in the training set, Cycle-GAN (Isola et al., 2018) is adopted to further augment the image dataset upon traditional methods. CycleGAN algorithm can realize image style transfer by learning two different types of images. In other words, there are now two image sample spaces $X$ and $Y . X$ represents the image space of healthy apples, and $Y$ represents the image space of diseased apples. The image in space $X$ is expected to be converted to an image in space $Y$. The Cycle-GAN algorithm transforms the image $x$ in space $X$ into the image $F(x)$ in space $Y$ through the generator $F$. In order to determine whether the generated image $F(x)$ is an image in space $Y$, the Cycle-GAN algorithm imports a discriminator $D_{Y}$, which, combined with the generator $F$, constitutes an adversarial neural network, as shown in Figure 2. Finally, by learning the image features of healthy and diseased apples, the generator converts the healthy apple image into a diseased apple image. The discriminator determines that the generated image is in the diseased apple image dataset, which completes the image style conversion.

To remedy the insufficient number of gray spot and serious cedar apple rust images in apple leaf disease dataset, rotation and brightness transformation were adopted to augment the original image. According to the lack of diseased apple fruit images, brightness transformation, rotation transformation and mirror transformation were first exploited to generate abundant images. Subsequently, the Cycle-GAN technique was utilized to perform feature learning on general anthracnose, serious anthracnose, and ring rot images, then generate these three types of lesions on healthy apple images. After image style transformation, the generated images were provided to disease experts, who selected 500 images of each type of disease that could be employed as training samples. These generated images were added to the dataset. The dataset composition after image augmentation is shown in Table 1.

\subsection{Multi-Scale Dense Classification Network}

Deep learning network is an effective method to achieve target classification. In recent years, LeNet (Lecun et al., 1998), AlexNet (Krizhevsky et al., 2012), VGG (Simonyan and Zisserman, 2015), GoogLeNet (Szegedy et al., 2015), DenseNet (Huang et al., 2017), Inception (Szegedy et al., 2016), etc. have been widely used in target classification. Due to the complex backgrounds of apple disease images, the image features of different diseases are similar, and the distinction between different grades of the same disease is inconspicuous. Therefore, it is necessary to further improve the performance of the existing deep learning networks. In general, increasing the number of network layers is employed to improve the fitting ability of the network. However, with the deepening of the network layers, the image features of the bottom layers are gradually lost. This results in the inadequate usages of image features. Therefore, when a network reaches a certain depth, increasing the number of network layers cannot continue to improve its capability.

As the Inception series classification networks have shown superior performance on multiple classification tasks, the stateof-the-art neural networks Inception-V4 and Inception-ResNetV2 (Szegedy et al., 2016) were adopted as the backbone in this paper. The idea of Multi-scale connection and DenseNet was introduced to construct new network models, achieving the purpose of promoting feature reuse and improving network performance.

\subsubsection{Inception-V4 and Inception-ResNet-V2}

Inception-V4 and Inception-ResNet-V2 networks were optimized on the basis of Inception-V3 (Szegedy et al., 2016). The network structure of Inception is shown in Figure 3, where Stem is the basic structure of feature extraction, which consists of multiple convolution and pooling operations. The Inception-X module in the backbone learns image features through the feature transfer architecture of several parallel structures, which has a higher feature utilization rate than the previous Inception versions. One basic mechanism of the Inception module is shown as Figure 4. The entire Inception structure is strung together by 

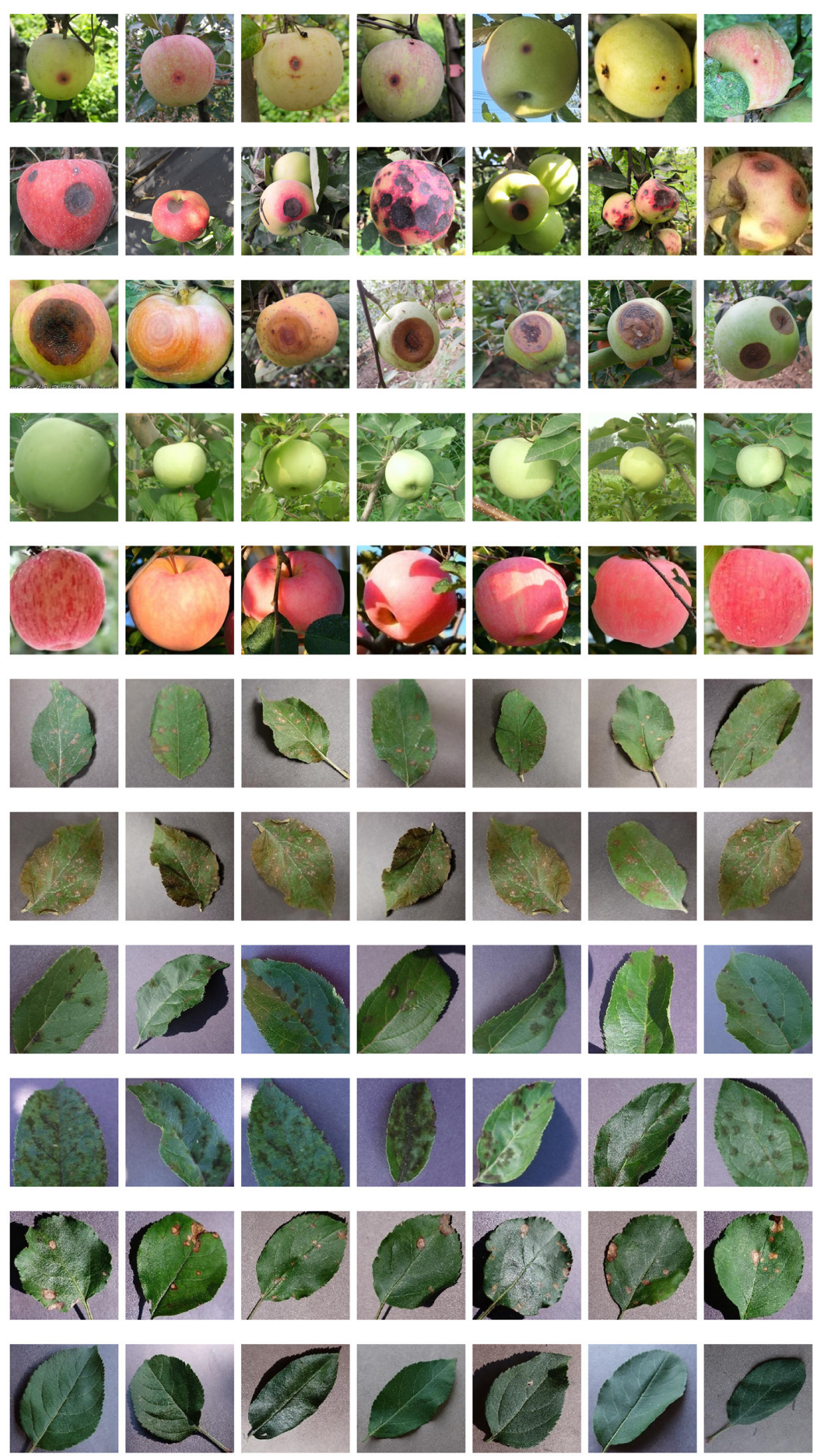

FIGURE 1 | Some images of the dataset adopted in this paper, from top to bottom, are general anthracnose, serious anthracnose, ring rot, healthy green apple, healthy red apple, general cedar apple rust, serious cedar apple rust, general apple scab, serious apple scab, apple gray spot, healthy apple leaf. 
TABLE 1 | Categories, labels, and quantity of images in the dataset.

\begin{tabular}{|c|c|c|c|}
\hline Category & Label & Size of original datasets & Size of augmented datasets \\
\hline Healthy apple leaf & 0 & 1,354 & 1,354 \\
\hline General apple scab & 1 & 1,205 & 1,205 \\
\hline Serious apple scab & 2 & 1,026 & 1,026 \\
\hline Apple gray spot & 3 & 488 & 976 \\
\hline General cedar apple rust & 4 & 972 & 972 \\
\hline Serious cedar apple rust & 5 & 460 & 1,380 \\
\hline Healthy green apple fruit & 6 & 1,360 & 1,360 \\
\hline Healthy red apple fruit & 7 & 1,360 & 1,360 \\
\hline General anthracnose & 8 & 78 & 1,014 \\
\hline Serious anthracnose & 9 & 87 & 1,044 \\
\hline Ring rot & 10 & 93 & 1,023 \\
\hline
\end{tabular}

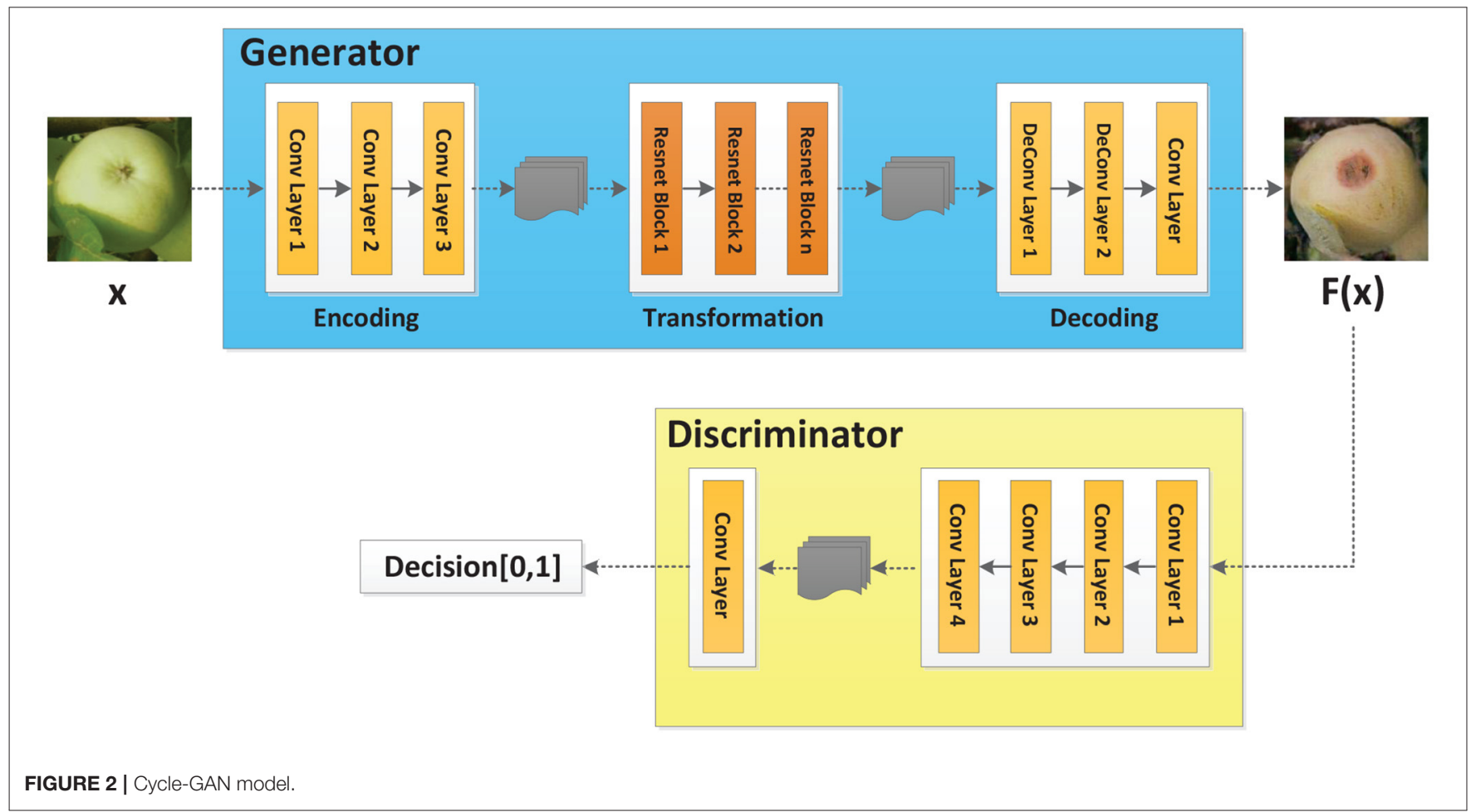

several Inception modules. There are two main contributions of the Inception structure. One is to use $1 \times 1$ convolution to raise and lower the dimension. The other is to convolve and aggregate on multiple dimensions at the same time. Inception-ResNet-V2 introduces the ResNet module over inception-V4, which further improves the performance of the network.

\subsubsection{DenseNet}

In order to make better use of the image features, DenseNet (Huang et al., 2017) structure was introduced into the networks. DenseNet realizes the concatenation of features in different depths by establishing dense connections between the front layers and the back layers, thereby achieving feature reuse.
Each Inception-(ResNet-)X module in the Inception-X layer and Inception-ResNet-X layer is called an Inception block. The four Inception-A modules in Inception-V4 are taken as an example, demonstrated in Figure 5. The output of the first Inception block is processed by the $\mathrm{H} 1$ function and then used as the input of the second Inception block. The output of the second Inception block is cascaded with the output of the previous block after $\mathrm{H} 2$ function operation. Their concatenation is the input of the third Inception block. The output of the third Inception block is cascaded with the output of the previous two Inception blocks after $\mathrm{H} 3$ function and then adopted as the input of the fourth Inception block. Finally, the outputs of all Inception blocks are spliced as the input of the subsequent network. In this paper, Hi stands for non-linear conversion function, which 


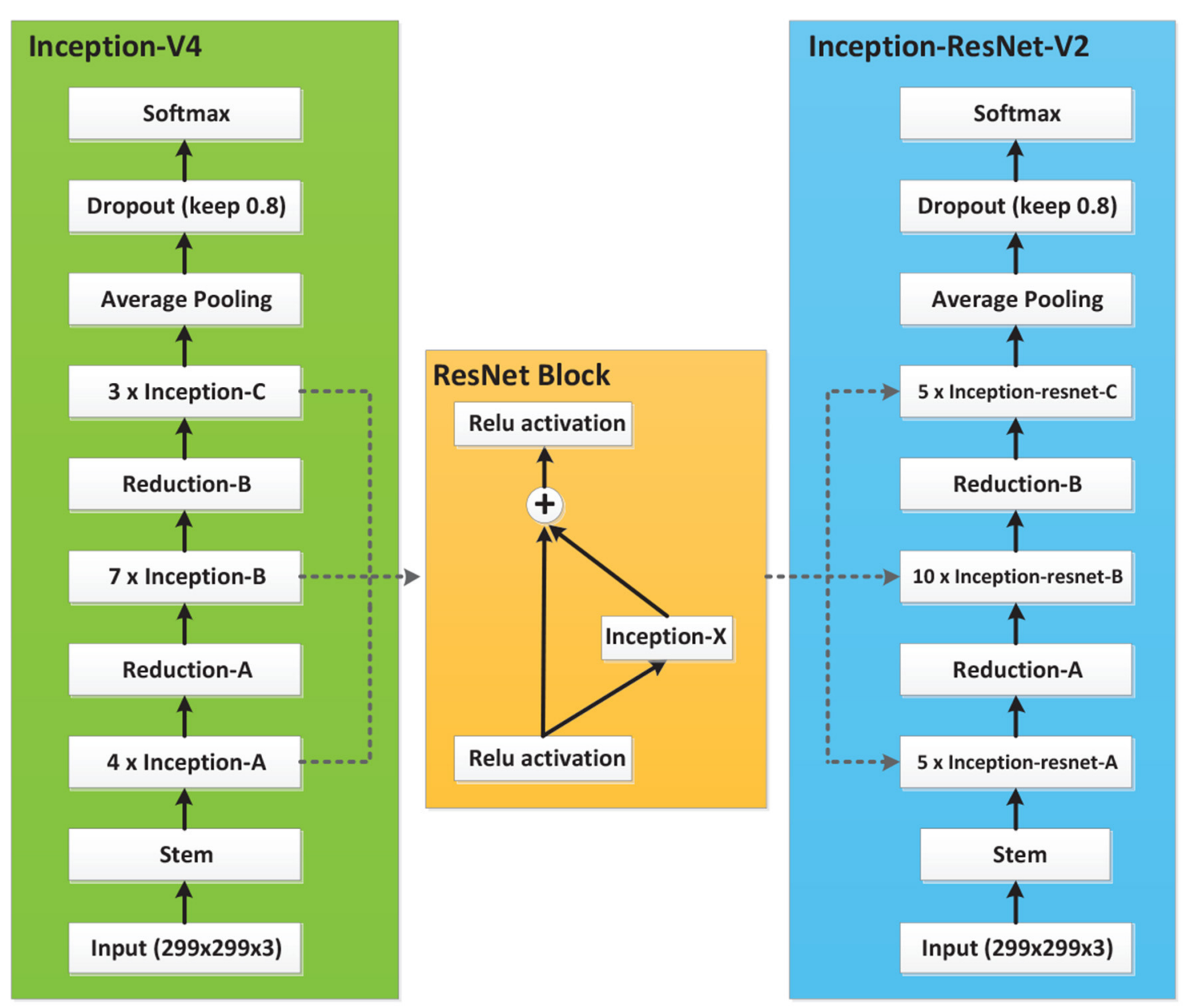

FIGURE 3 | Network structures of Inception-V4 and Inception-ResNet-V2.

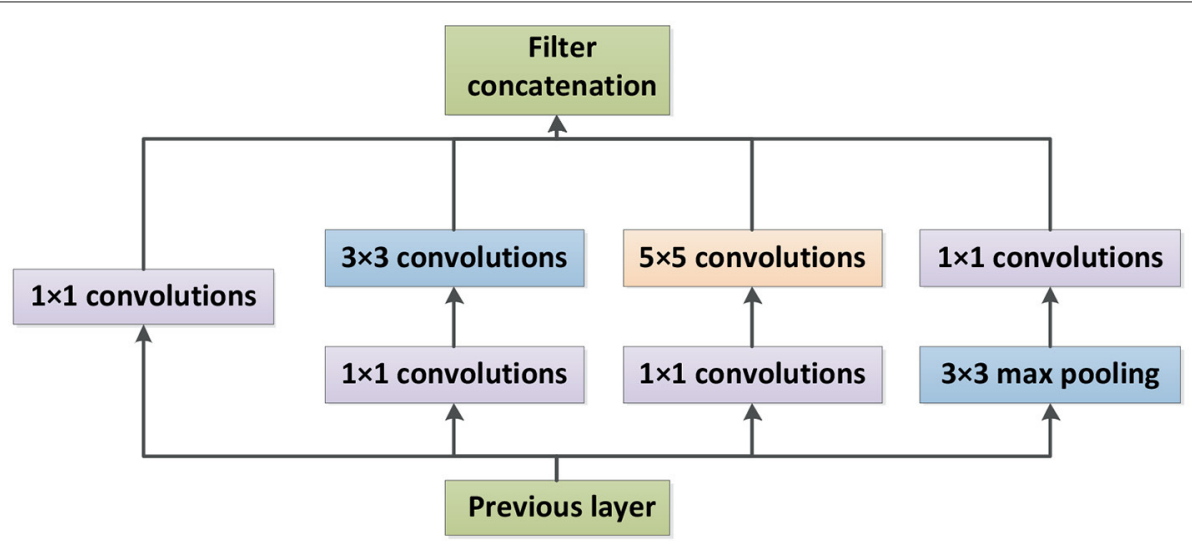

FIGURE 4 | Inception module.

is a combination structure of batch normalization (BN), rectified linear units (RELU), and convolution (Conv), named BN-ReLUConv $(1 \times 1)$-BN-ReLU-Conv $(3 \times 3)$.

\subsubsection{Multi-Scale Connection}

On the basis of the Dense Inception block, a Multi-scale concatenation structure was designed to splice the different 


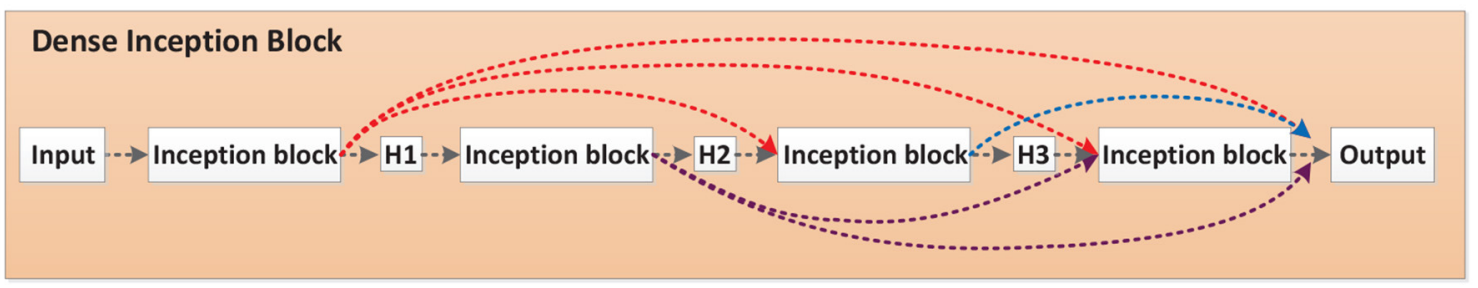

FIGURE 5 | Dense Inception block.
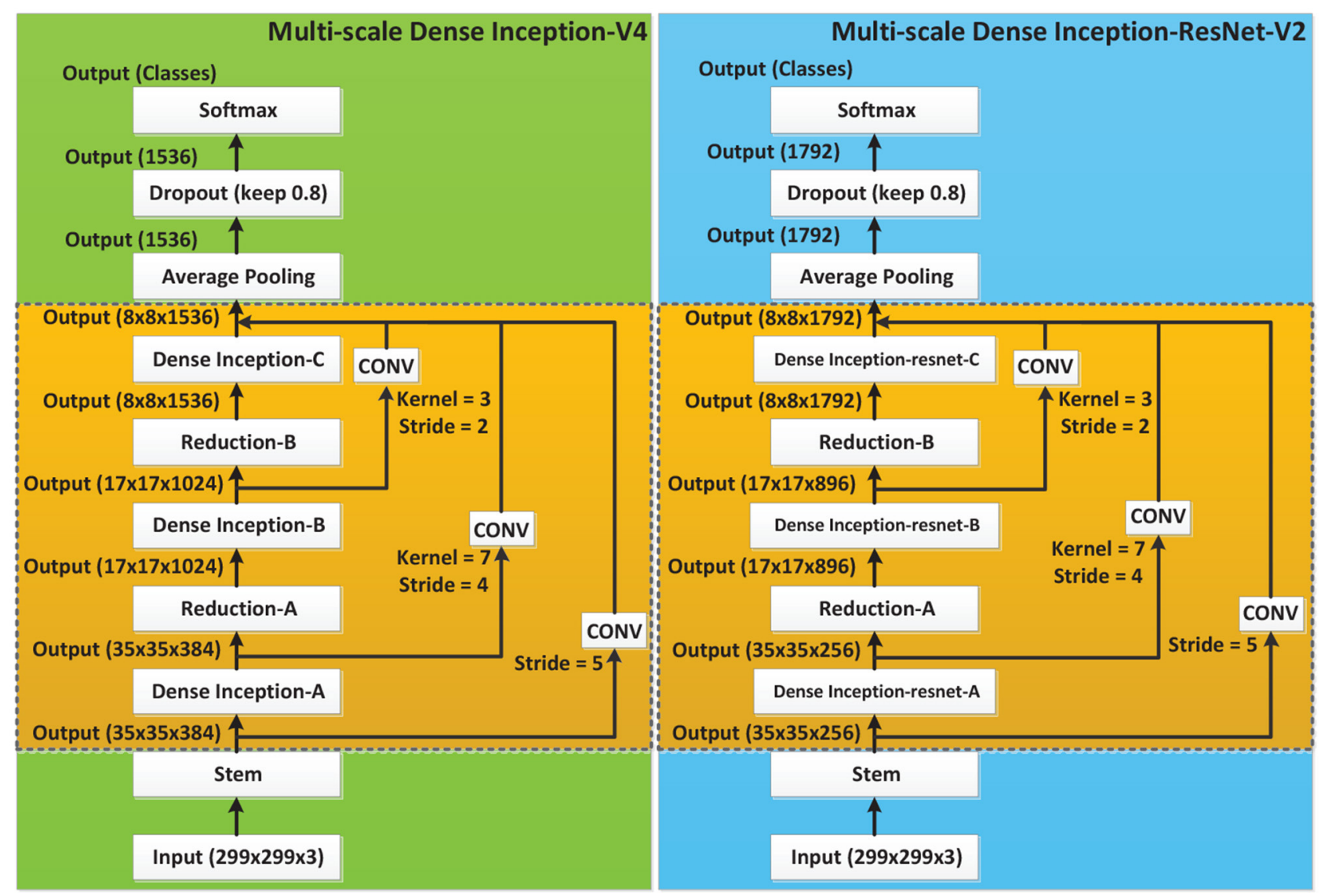

FIGURE 6 | Multi-scale Dense neural networks.

depths of the network, as shown in Figure 6. In the Multi-scale concatenation network model, the output of the Stem block is convolved and spliced with the output of each Dense Inception block. The concatenation result is sent to the average pooling layer as the input. Multi-scale connection enables network features of different depths to function in the final training, which further improves the utilization of bottom layer features.

\subsubsection{Details of Model Training}

In this paper, the image dataset composition is shown in Table 1. In order to verify the performance of the deep learning models, 350 images of each disease were randomly selected from the augmented image dataset as the test set, and the remaining images as the training set. All diagnostic models were trained and tested on the IW4210-8G server. In the training process of Multiscale Dense Inception-V4 and Multi-scale Dense InceptionResNet-V2, the number of epoches was set to 4,000, the minimum batch was set to 16 , the dropout was set to 0.8 , the learning rate was set to 0.0001 and the decay rate was set to 0.95 . The cross entropy function was used as the loss function as follows.

$$
\text { Loss }=-\frac{1}{N} \sum_{i=1}^{N} \sum_{c=1}^{M} y_{i c} \log \left(p_{i c}\right)
$$




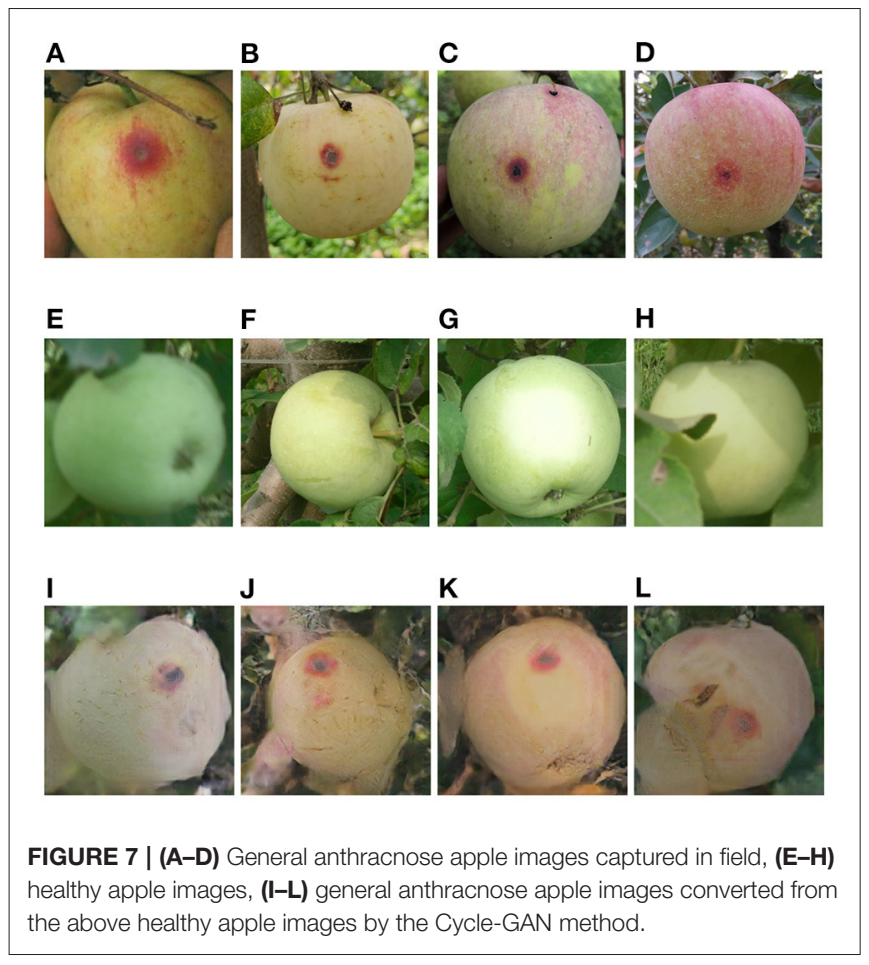

where $N$ is the number of training samples. $M$ is the number of categories. $y_{i c}$ is a symbolic function that is 1 when the true class of sample $i$ is $c$, and 0 otherwise. $p_{i c}$ is the prediction probability of sample $i$ belonging to category $c$.

\section{EXPERIMENTS AND DISCUSSION}

\subsection{Evaluation of Cycle-GAN Method}

The Cycle-GAN method is introduced on the basis of traditional image augmentation methods to expand the image dataset. Some of the healthy and diseased apple images captured in the field and the diseased apple images generated by Cycle-GAN are shown in Figures 7-9. It can be seen that the generated diseased apple images are similar to the real images, and the disease characteristics are well-preserved. These images can be added to the dataset as the training samples. As described in section 2.1.2, these qualified images are selected from the generated images by experts. Images that can meet the requirements account for $73.1 \%$ of all generated images.

In order to better evaluate the Cycle-GAN method, two training sets were compared. One is a training set that contains images generated by Cycle-GAN. The other is the dataset that only adopts traditional augmentation techniques, such as brightness transformation, rotation transformation, and mirror transformation. The two training sets were employed to train the classification models, and the test results are shown in Table 2. According to the experimental results, Cycle-GAN method can generate new training samples in the super-pixel space and improve the richness of the training dataset more effectively than traditional image augmentation methods.

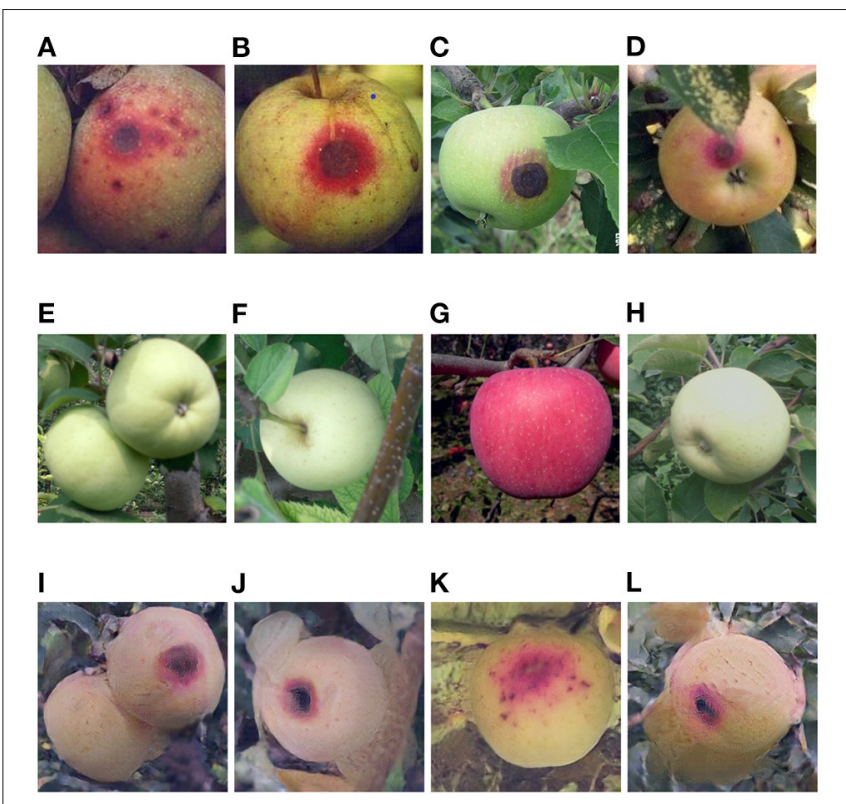

FIGURE 8 | (A-D) Serious anthracnose apple images captured in field, (E-H) healthy apple images, (I-L) serious anthracnose apple images converted from the above healthy apple images by the Cycle-GAN method.

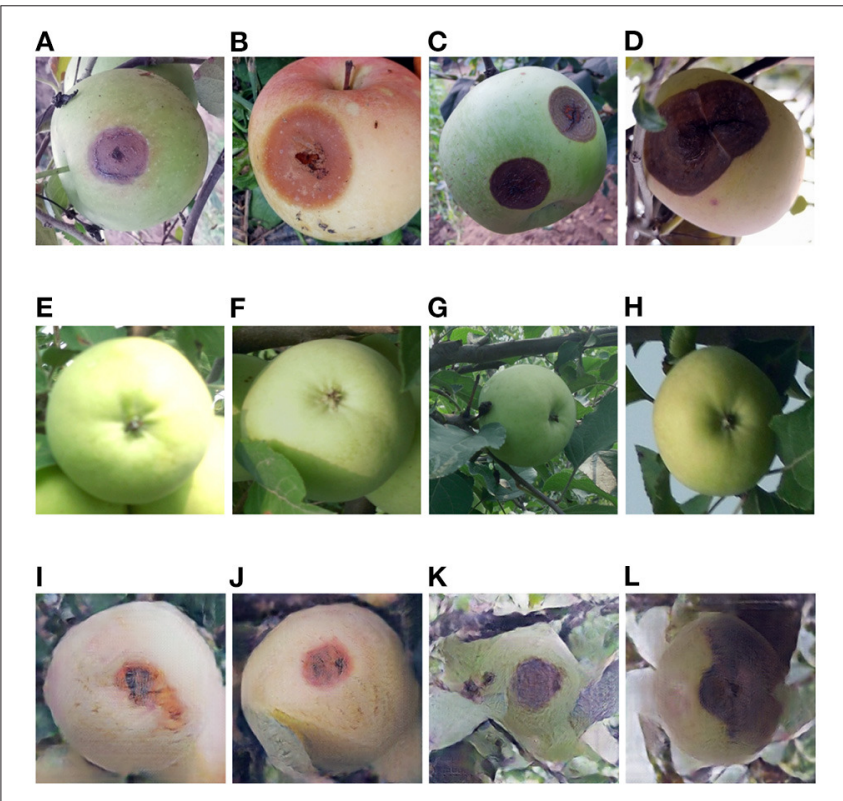

FIGURE 9 | (A-D) Ring rot apple images captured in field, (E-H) healthy apple images, (I-L) ring rot apple images converted from the above healthy apple images by the Cycle-GAN method.

\subsection{Comparison of Different Models}

In order to evaluate the performance of the proposed Multiscale Dense architecture, Inception-V4, Inception-ResNet-V2, Multi-scale Dense Inception-V4, Multi-scale Dense InceptionResNet-V2, LeNet, AlexNet, VGG, and Densenet-121 were 
TABLE 2 | Test results on different datasets.

\begin{tabular}{|c|c|c|c|c|c|}
\hline Model & Training set & Accuracy (\%) & Precision (\%) & Sensitivity (\%) & F1 score (\%) \\
\hline Multi-scale Dense Inception-V4 & With Cycle-GAN & 94.31 & 94.36 & 94.28 & 94.32 \\
\hline Multi-scale Dense Inception-ResNet-V2 & With Cycle-GAN & 94.74 & 94.71 & 95.03 & 94.86 \\
\hline Multi-scale Dense Inception-V4 & Without Cycle-GAN & 93.43 & 93.71 & 94.14 & 93.92 \\
\hline Multi-scale Dense Inception-ResNet-V2 & Without Cycle-GAN & 93.77 & 93.88 & 94.76 & 94.32 \\
\hline
\end{tabular}

TABLE 3 | Comparison of different models.

\begin{tabular}{|c|c|c|c|c|c|}
\hline Model & Accuracy (\%) & Precision (\%) & Sensitivity (\%) & Size (M) & Training time (hours) \\
\hline Inception-V4 & 92.72 & 92.61 & 94.89 & 176 & 2.32 \\
\hline Inception-ResNet-V2 & 93.37 & 92.65 & 95.37 & 226 & 1.95 \\
\hline Multi-scale Dense Inception-V4 & 94.31 & 94.36 & 94.28 & 201 & 2.58 \\
\hline Multi-scale Dense Inception-ResNet-V2 & 94.74 & 94.71 & 95.03 & 255 & 2.11 \\
\hline LeNet & 85.21 & 83.66 & 88.39 & 13 & 0.64 \\
\hline AlexNet & 92.04 & 91.75 & 92.83 & 60 & 1.79 \\
\hline VGG & 92.45 & 92.32 & 92.54 & 472 & 1.65 \\
\hline Densenet-121 & 93.68 & 93.66 & 95.12 & 46 & 3.29 \\
\hline
\end{tabular}

compared. All classification models were trained and tested on the IW4210-8G server. The training parameters of all models were unified. The experimental results of the eight models are shown in Table 3. It can be seen from Table 3 that there is no direct relationship between the training time and the size of the model, and the introduction of feature layer connection methods such as ResNet can accelerate the convergence of the neural network. The introduction of the Multi-scale connection and DenseNet has significantly improved the performance of Inception-V4 and Inception-ResNet-V2 models. The Multiscale Dense Inception-ResNet-V2 model achieves the best accuracy, surpasses the Densenet-121 model, and realizes the state-of-the-art performance.

\subsection{Diagnosis of Different Diseases}

In this paper, two models are proposed to classify images of 11 categories. The diagnosis results of different diseases are shown in Table 4. Accuracyl is the diagnosis result of the Multi-scale Dense Inception-V4 model, and Accuracy2 is the diagnosis result of the Multi-scale Dense Inception-ResNet-V2 model. It can be seen from the results in Table $\mathbf{4}$ that healthy apple leaf and healthy apple fruits have the highest diagnostic accuracy. Because the distinction between general disease and serious diseases is not obvious, the accuracy of the diagnosis result is affected. In addition, the image features of apple gray spot and cedar apple rust have a certain similarity, so a few inaccurate diagnosis results are given among the test samples. However, combining the results of various categories, the two new models have shown superior results and can meet the diagnosis requirements.

\subsection{Practical Application Scenarios}

In order to apply the disease diagnosis model in the actual scenario, a related disease management software is developed. The software consists of a human-computer interface on web page and a cloud data processing system.
TABLE 4 | The diagnosis results of two models for different diseases.

\begin{tabular}{lcc}
\hline Disease & Accuracy1 (\%) & Accuracy2 (\%) \\
\hline Healthy apple leaf & 95.63 & 95.82 \\
General apple scab & 93.18 & 93.21 \\
Serious apple scab & 93.45 & 93.79 \\
Apple gray spot & 92.11 & 92.27 \\
General cedar apple rust & 92.80 & 92.65 \\
Serious cedar apple rust & 93.12 & 93.86 \\
Healthy green apple fruit & 96.34 & 96.73 \\
Healthy red apple fruit & 96.22 & 96.75 \\
General anthracnose & 94.27 & 94.77 \\
Serious anthracnose & 94.15 & 94.45 \\
Ring rot & 93.97 & 94.38 \\
Overall & 94.31 & 94.74 \\
\hline
\end{tabular}

The Multi-scale Dense Inception-ResNet-V2 classification model is deployed in the cloud data processing system. In practice, the orchard staff can upload the disease images collected in field through the human-computer interface. The cloud system obtains the diagnosis results by processing the uploaded images and gives feedback to the humancomputer interface. Some of the diagnostic results are shown in Figure 10. In practical application scenarios, 54 disease images collected in orchards were diagnosed, and the diagnostic accuracy was $94.44 \%$. With the continuous expansion of the disease image datasets, the diagnosis model can also identify more kinds of diseases, which will further reflects the generalization ability. 


\section{A}

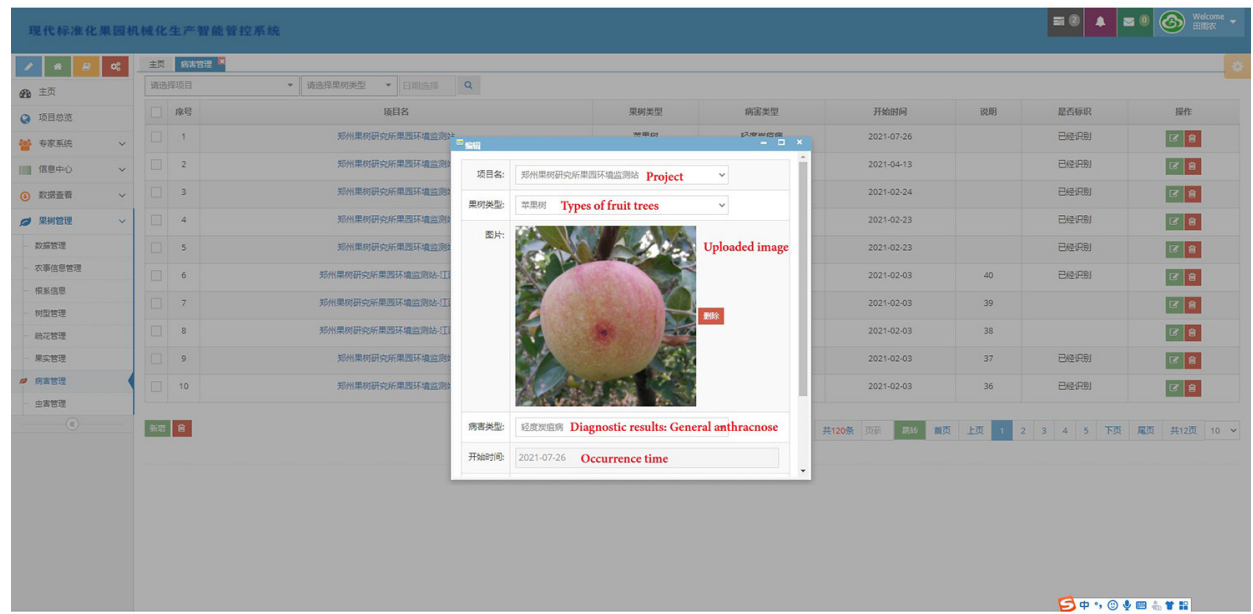

B

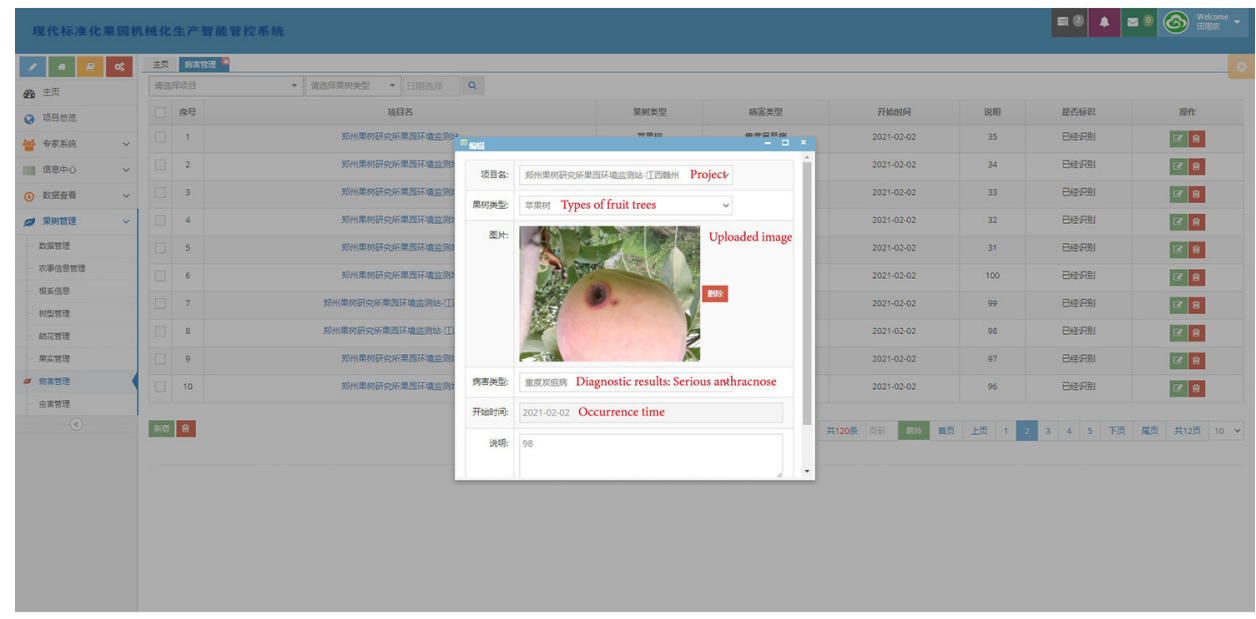

\section{C}

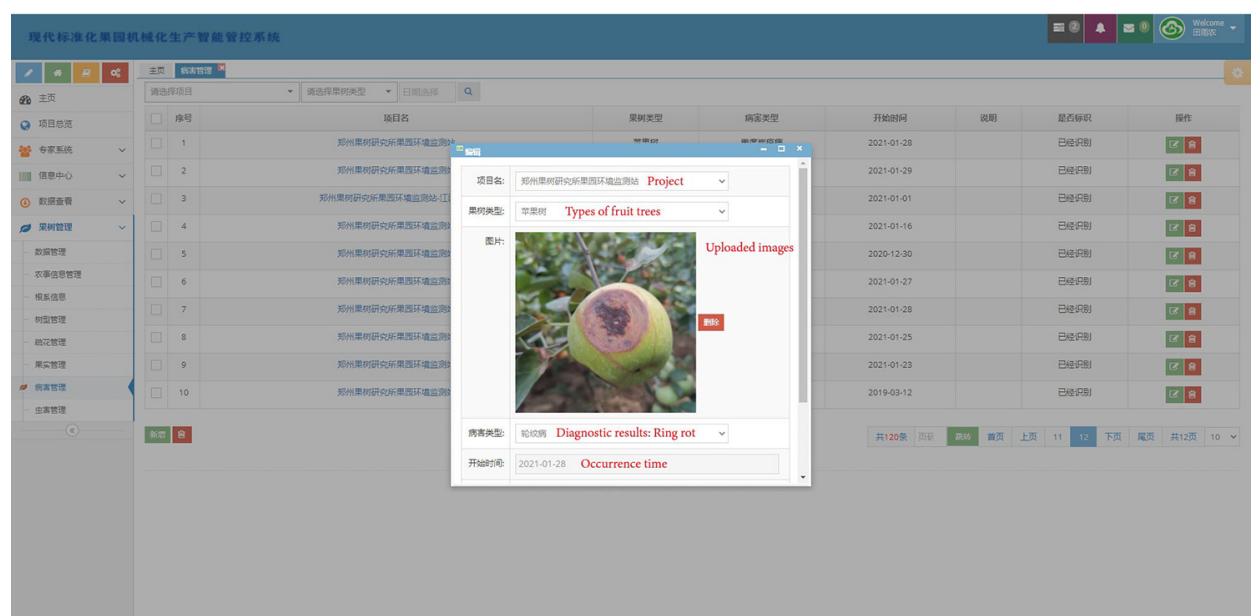

FIGURE 10 | Several diagnosis results in practical application scenarios. (A) General anthracnose, (B) serious anthracnose, (C) ring rot. 


\section{CONCLUSIONS}

A Multi-scale Dense network architecture is proposed in this paper to diagnose images of 11 categories including healthy apple leaves and fruits and typical diseased apple leaves and fruits. The diagnosis results have reached the state-of-the-art performance. The detailed work is summarized as follows.

(1) Aiming at the problem of insufficient image data for general anthracnose, serious anthracnose, and ring rot, the Cycle-GAN method is adopted to learn the image features of healthy apples and diseased apples, and generates different categories of the lesions on the surface of healthy apple images. Experiments have verified that the generated images have greatly enriched the image dataset. The Cycle-GAN technique outperforms traditional image augmentation methods.

(2) The DenseNet and Multi-scale connection method are employed to improve the existing deep learning models, and the Multi-scale Dense Inception-V4 and Multi-scale Dense Inception-ResNet-V2 classification models are proposed. Experiments have shown that the detection accuracy of the two proposed models have reached 94.31 and $94.74 \%$, respectively, which not only improves the classification results of the original models, but also achieves the state-of-the-art diagnosis performance.

\section{REFERENCES}

Amara, J., Bouaziz, B., and Algergawy, A. (2017). "A deep learning-based approach for banana leaf diseases classification,” in Datenbanksysteme für Business, Technologie und Web (BTW 2017), 17. Fachtagung des GI-Fachbereichs, Datenbanken und Informationssysteme (DBIS) (Stuttgart), 79-88.

Bargoti, S., and Underwood, J. (2016). "Deep fruit detection in orchards," in 2017 IEEE International Conference on Robotics and Automation (ICRA) (Singapore), 1-8. doi: 10.1109/ICRA.2017.7989417

Dyrmann, M., Karstoft, H., and Midtiby, H. (2016). Plant species classification using deep convolutional neural network. Biosyst. Eng. 151, 72-80. doi: 10.1016/j.biosystemseng.2016.08.024

Huang, G., Liu, Z., V. D.,Maaten, L., and Weinberger, K. (2017). "Densely connected convolutional networks," in IEEE Conference on Computer Vision and Pattern Recognition (Honolulu, HI), 2261-2269. doi: 10.1109/CVPR.2017.243

Isola, P., Zhu, J., Zhou, T., and Efros, A. (2018). "Image-to-image translation with conditional adversarial networks," in 2017 IEEE Conference on Computer Vision and Pattern Recognition (CVPR) (Honolulu, HI), 1063-6919. doi: 10.1109/CVPR.2017.632

Jia, Y., Shelhamer, E., Donahue, J., Karayev, S., Long, J., Girshick, R., et al. (2014). "Caffe: convolutional architecture for fast feature embedding," in MM 2014 - Proceedings of the 2014 ACM Conference on Multimedia (Orlardo, Forida). doi: 10.1145/2647868.2654889

Jiang, P., Chen, Y., Liu, B., He, D., and Liang, C. (2019). Real-time detection of apple leaf diseases using deep learning approach based on improved convolutional neural networks. IEEE Access. 7, 59069-59080. doi: 10.1109/ACCESS.2019.2914929

Khanna, A., and Kaur, S. (2019). Evolution of Internet of Things (IoT) and its significant impact in the field of Precision Agriculture. Comput. Electron. Agric. 157, 218-231. doi: 10.1016/j.compag.2018. 12.039
In future work, we will collect more images of different types of apple diseases and analyze the image characteristics of different diseases. The automatic diagnosis ability will be further enhanced through dataset expansion and model improvement.

\section{DATA AVAILABILITY STATEMENT}

The original contributions presented in the study are included in the article/Supplementary Material, further inquiries can be directed to the corresponding author/s.

\section{AUTHOR CONTRIBUTIONS}

YT was responsible for methodological research and paper writing. EL and MT guided the research ideas and framework of the paper. ZL was responsible for the research of disease data augmentation. $\mathrm{XH}$ provided the raw data for the experiments. All authors contributed to the article and approved the submitted version.

\section{FUNDING}

This work was supported by the National Key Research and Development Plan (2017YFC0806501), National Natural Science Foundation (U1713224 and 61973300), and the Science and Technology Innovation Project of Beijing (Z181100003818007).

Krizhevsky, A., Sutskever, I., and Hinton, G. (2012). "Imagenet classification with deep convolutional neural networks," in NIPS'12 - Proceedings of the 25th International Conference on Neural Information Processing Systems (Red Hook, NY), 84-90. doi: 10.1145/3065386

Lecun, Y., Bottou, L., Bengio, Y., and Haffner, P. (1998). Gradient-based learning applied to document recognition. Proc. IEEE 86, 2278-2324. doi: 10.1109/5.726791

Mohanty, S., Hughes, D., and Salathé, M. (2016). Using deep learning for image-based plant disease detection. Front. Plant Sci. 7:1419. doi: 10.3389/fpls.2016.01419

Muangprathub, J., Boonnam, N., Kajornkasirat, S., Lekbangpong, N., Wanichsombat, A., and Nillaor, P. (2019). IoT and agriculture data analysis for smart farm. Comput. Electron. Agric. 156, 467-474. doi: 10.1016/j.compag.2018.12.011

Omrani, E., Khoshnevisan, B., Shamshirband, S., Saboohi, H., Anuar, N., and Nasir, M. (2014). Potential of radial basis function-based support vector regression for apple disease detection. Measurement 55, 512-519. doi: 10.1016/j.measurement.2014.05.033

Patricio, D., and Rieder, R. (2018). Computer vision and artificial intelligence in precision agriculture for grain crops: a systematic review. Comput. Electron. Agric. 153, 69-81. doi: 10.1016/j.compag.2018.08. 001

Rumpf, T., Mahlein, A., Steiner, U., Oerke, A., Dehne, H., and Plümer, L. (2010). Early detection and classification of plant diseases with Support Vector Machines based on hyperspectral reflectance. Comput. Electron. Agric. 74, 91-99. doi: 10.1016/j.compag.2010.06.009

Sankaran, S., Mishra, A., Ehsani, R., and Davis, C. (2010). A review of advanced techniques for detecting plant diseases. Comput. Electron. Agric. 72, 1-13. doi: 10.1016/j.compag.2010.02.007

Shen, T., Gou, C., He, Z., and Chen, W. (2019). Learning from adversarial medical images for X-ray breast mass segmentation. Comput. Methods Prog. Biomed. 180:105012. doi: 10.1016/j.cmpb.2019.105012 
Simonyan, K., and Zisserman, A. (2015). "Very deep convolutional networks for large-scale image recognition," in 2015 International Conference on Learning Representations (ICLR) (San Diego, California), 1-14. arXiv preprint arXiv:1409.1556.

Sladojevic, S., Arsenovic, M., Anderla, A., Culibrk, D., and Stefanovic, D. (2016). Deep neural networks based recognition of plant diseases by leaf image classification. Comput. Intell. Neurosci. 2016, 1-11. doi: 10.1155/2016/3289801

Szegedy, C., Ioffe, S., Vanhoucke, V., and Alemi, A. (2016). "Inception-v4, inception-resnet17 and the impact of residual connections on learning." in AAAI'17:- Proceedings of the Thirty-First AAAI Conference on Artificial Intelligence (San Francisco, California). doi: 10.5555/3298023.3298188

Szegedy, C., Liu, W., Jia, Y., Sermanet, P., Reed, S., Anguelov, D., et al. (2015). "Going deeper with convolutions," in 2015 The IEEE Conference on Computer Vision and Pattern Recognition (CVPR) (Boston, MA), 1-9. doi: 10.1109/CVPR.2015.7298594

Tan, W., Zhao, C., and $\mathrm{Wu}, \mathrm{H}$. (2016). Intelligent alerting for fruit-melon lesion image based on momentum deep learning. Multimedia Tools Appl. 75, 16741-16761. doi: 10.1007/s11042-015-2940-7

Tian, Y., Li, E., Yang, L., and Liang, Z. (2018). "An image processing method for green apple lesion detection17 in natural environment based on GA-BPNN and SVM," in 2018 IEEE International Conference on Mechatronics and Automation (ICMA) (Changchun, Shenyang), 1210-1215. doi: 10.1109/ICMA.2018.8484624

Tian, Y., Yang, G., Wang, Z., Li, E., and Liang, Z. (2019a). Detection of apple lesions in orchards based on deep learning methods of CycleGAN and YOLOV3dense. J. Sens. 2019:7630926. doi: 10.1155/2019/7630926

Tian, Y., Yang, G., Wang, Z., Li, E., and Liang, Z. (2020). Instance segmentation of apple flowers using the improved mask R-CNN model. Biosyst. Eng. 193, 264-278. doi: 10.1016/j.biosystemseng.2020.03.008

Tian, Y., Yang, G., Wang, Z., Wang, H., Li, E., and Liang, Z. (2019b). Apple detection during different growth stages in orchards using the improved YOLO-V3 model. Comput. Electron. Agric. 157, 417-426. doi: 10.1016/j.compag.2019.01.012

Zarifneshat, S., Rohani, A., Ghassemzadeh, H., Sadeghi, M., Ahmadi, E., and Zarifneshat, M. (2012). Predictions of apple bruise volume using artificial neural network. Comput. Electron. Agric. 82, 75-86. doi: 10.1016/j.compag.2011.12.015

Zhang, J., Huang, Y., Pu, R., Gonzalez-Moreno, P., Yuan, L., Wu, K., et al. (2019). Monitoring plant diseases and pests through remote sensing technology: a review. Comput. Electron. Agric. 165:104943. doi: 10.1016/j.compag.2019.104943

Zhang, S., Zhang, S., Zhang, C., Wang, X., and Shi, Y. (2019). Cucumber leaf disease identification with global pooling dilated convolutional neural network. Comput. Electron. Agric. 162, 422-430. doi: 10.1016/j.compag.2019.03. 012

Zhong, Y., and Zhao, M. (2020). Research on deep learning in apple leaf disease recognition. Comput. Electron. Agric. 168:105146. doi: 10.1016/j.compag.2019.105146

Zou, X., Zhao, J., Li, Y., and Mel, H. (2010). In-line detection of apple defects using three color cameras system. Comput. Electron. Agric. 70, 129-134. doi: 10.1016/j.compag.2009.09.014

Conflict of Interest: The authors declare that the research was conducted in the absence of any commercial or financial relationships that could be construed as a potential conflict of interest.

Publisher's Note: All claims expressed in this article are solely those of the authors and do not necessarily represent those of their affiliated organizations, or those of the publisher, the editors and the reviewers. Any product that may be evaluated in this article, or claim that may be made by its manufacturer, is not guaranteed or endorsed by the publisher.

Copyright (c) 2021 Tian, Li, Liang, Tan and He. This is an open-access article distributed under the terms of the Creative Commons Attribution License (CC BY). The use, distribution or reproduction in other forums is permitted, provided the original author(s) and the copyright owner(s) are credited and that the original publication in this journal is cited, in accordance with accepted academic practice. No use, distribution or reproduction is permitted which does not comply with these terms. 